\title{
A Robust Medical Image Authentication Technique using QR Code and DWT
}

\author{
Sirshendu Hore \\ Asst. Professor Dept. of CSE, \\ Hooghly Engineering \& \\ Technology College, Hooghly, \\ West Bengal, India
}

\author{
Tanmay Bhattacharya \\ Asst. Professor Dept. of IT, \\ JIS College of Engineering, \\ Kalyani, West Bengal, India
}

\author{
S. R. Bhadra Chaudhuri \\ Professor, Dept of E\&TCE, \\ Bengal Engineering and \\ Science University, Shibpur, \\ West Bengal, India
}

\begin{abstract}
Image authentication plays a vital role in safeguarding the originality as well as helps to retain the integrity of the data. It is also used as proof in case of content delivery. This paper proposes an efficient and effective medical image authentication technique by embedding information of a patient and doctor through corresponding $\mathrm{QR}$ codes in selected DWT sub-band of that image. At the receiver end QR codes are extracted and information containing in the $\mathrm{QR}$ codes are retrieved using Smart Phone or through standard QR Code Reader.
\end{abstract}

\section{General Terms}

Image Authentication, QR Code, Smart Phone

\section{Keywords}

DWT, QR Code, PSNR, Medical Imaging

\section{INTRODUCTION}

Image authentication helps to verify the uniqueness of an image by detecting malicious skillful alteration. Thus through image authentication we maintain image originality. The most vital issue is to develop a robust image authentication technique that helps to protect the integrity of medical image against different attacks. The two most popular methods used for accomplishing the authenticity of digital images are: A digital signs on the image using a digital signature [1], or embedding a secret code in the image [2].

Through data hiding sensitive data are hidden within a medical message or image as secret or encrypted data and extractions of such data are done at its destination. Anyone except the sender and the receiver viewing the message or images will fail to know that it contains secret/encrypted data. [3, 4 and 5]

Spatial domain LSB [6] insertion is a very simple and common approach for embedding information in an image. The constraint of this approach is vulnerability to every minor image manipulation. Thus conversion of image from one format to another format and vice versa could annihilate secret information in LSBs. Through statistical analysis like histogram analysis, watermarks can be easily detected. The technique applied in spatial domains involves replacement of $\mathrm{N}$ least significant bit of each pixel of a container image with the data of a secret message. As $\mathrm{N}$ increases Watermarkedimage gets destroyed. In frequency domain Discrete Cosine Transformation (DCT) [7, 10] can be used for hiding secret information. DCT transformation suffers from blocking artifact. In DCT the pixels are grouped into $8 \times 8$ blocks and convert the pixel blocks into 64 DCT co-efficient. A modification of a single DCT co-efficient will affect all 64 image pixels in that block. Discrete Wavelet Transformation (DWT) based approach [8, 9, 13 and 14] is one of the modern approaches used for watermarking. In this approach the imperceptibility and distortion of the Watermarked image is acceptable and it can also successful in against several attacks.

Medical Imaging: Medical imaging consists of numerous diverse technologies that are used to observe the human body in order to monitor, diagnose, or treat medical situation. Medical imaging technology provides different information related to the area of the body being treated or studied. Thus through the use of medical imaging one can easily measure the effectiveness of medical treatment, injury, disease etc.

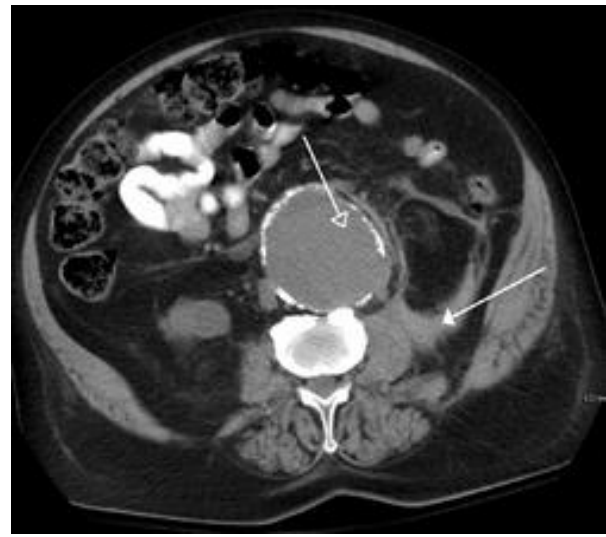

Fig 1: A CT scan image showing a ruptured abdominal aortic aneurysm.

QR Code: QR code, a new Jargon in today's world, stands for Quick Response code, is now becomes a trademark for business and all purpose use is a kind of two-dimensional matrix first designed to be used for automotive industry in Japan. A QR Code records information of an item in the form of a machine-readable label which is encoded and can be attached to that item. One may use four standardized modes of data such as alphanumeric, byte or binary, numeric and kanji to store or encoded information in a QR code. [12, 15] 

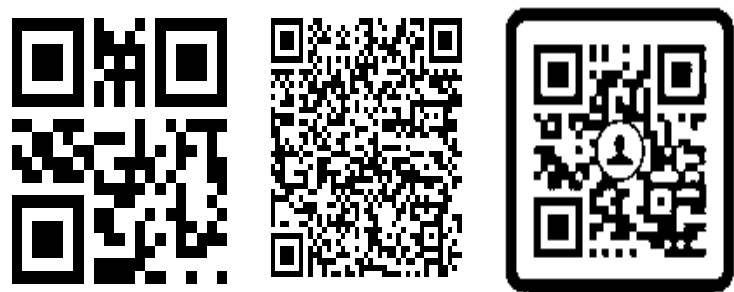

Fig 2: Different QR Code

The Length of QR Codes is 8 bits and they follow four error correction levels algorithm known as Reed-Solomon error correction algorithm. The Storage capacity decreases as the error correction level increases. Table 1 illustrated the four levels with their approximate error correction capability.

Table 1: Four levels of QR Code with their approximate error correction capability

\begin{tabular}{|c|c|}
\hline Level L (Low) & $\begin{array}{c}\text { 07\% of codeword's can be } \\
\text { restored. }\end{array}$ \\
\hline $\begin{array}{c}\text { Level M } \\
\text { (Medium) }\end{array}$ & $\begin{array}{c}15 \% \text { of codeword's can be } \\
\text { restored. }\end{array}$ \\
\hline $\begin{array}{c}\text { Level Q } \\
\text { Quartile) }\end{array}$ & $\begin{array}{c}25 \% \text { of codeword's can be } \\
\text { restored. }\end{array}$ \\
\hline Level H (High) & $30 \%$ of codeword's can be \\
restored.
\end{tabular}

Standards: There are several standards used for QR Code such as - "JIS X 0510", "ISO/IEC 18004:2000", "ISO/IEC 18004:2006" [11] etc.

QR Code Image Generator \& Extractor: It is used to generate QR code from Text, Contact, URL, SMS, Picture and .pdf while QR code extractor are used to retrieve that information from the QR Code

Advantage: The QR Code system has become popular outside the automotive industry due to its fast readability and greater storage capacity compared to standard UPC barcodes

Area of Application: Applications include item identification, product tracking, time tracking, general marketing, document management and much more. QR codes can be used in i-phone or i-pad also Nokia Symbian Belle, BlackBerry OS, Google's Android and Apple iOS devices, as well as Microsoft's Windows Phone operating system.

Limitation: A QR code can contain thousands of characters, but recommend size limit is up to 100 characters. As QR codes grow in size as more and more characters are stuffed in, it is observed that Smart phone camera resolution or Reader really can't handle such larger ones. Therefore if the size is about 100 characters or so, it seems likely that most smart phones or reader will have less trouble while reading QR code.

\section{Discrete Wavelet Transformation}

The wavelet transform describes a multi-resolution decomposition process in terms of expansion of an Image onto a set of wavelet basis functions. Discrete Wavelet Transformation has its own excellent space frequency localization property. The input image is divided into 4 nonoverlapping multi-resolution sub-bands by the filters, namely (LL1), (LH1), (HL1) and (HH1). The sub-band (LL1) is processed further to obtain the next coarser scale of wavelet coefficients, until some final scale " $\mathrm{N}$ " is reached. When "N" is reached, it will have $3 \mathrm{~N}+1$ sub-bands consisting of the multi-resolution sub-bands (LLN) and (LHX), (HLX) and (HHX) where "X" ranges from 1 until "N". Generally most of the Image energy is stored in these sub-bands.

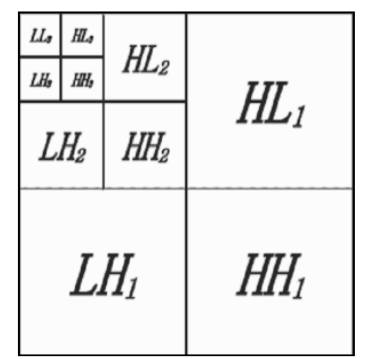

Fig 3: Three phase decomposition using DWT

The Forward Discrete Wavelet Transform is very suitable to identify the areas in the Medical image where a watermarked image can be embedded effectively due to its excellent spacefrequency localization properties. In particular, this property allows the exploitation of the masking effect of the human visual system such that if a DWT co-efficient is modified, it modifies only the region corresponding to that coefficient.

The embedding secret image in the lower frequency subbands (LLX) may degrade the image significantly, as generally most of the Image energy is stored in these subbands. Embedding in the low-frequency sub-bands, however, could increase robustness significantly. In contrast, the edges and textures of the image and the human eye are not generally sensitive to changes in the high frequency sub-bands (HHX). This allows the watermarked-image to be embedded without being perceived by the human eye. The compromise adopted by many DWT based algorithms, to achieve acceptable performance of imperceptibility and robustness, is to embed the secret image in the middle frequency sub-bands (LHX) or (HLX) and (HHX). The Haar wavelet is also the simplest possible wavelet. Haar wavelet is not continuous, and therefore not differentiable. This property can, however, be an advantage for the analysis of signals with sudden transitions

\section{PROPOSED ALGORITHM}

\subsection{QR Code Generations}

Two separate $\mathrm{QR}$ codes have been generated based on Patient's record and Doctor's record

\subsection{QR Code Embedding}

QR code embedding steps are as follows:

a. Original medical image is decomposed into four sub bands (LL2, LH2, HL2 and HH2) using DWT twice

b. Two QR codes are converted into two 1D image vectors separately. 
c. Two 2D Pseudo Noise (PN) sequences are generated from two session based keys.

d. HL2 and HH2 sub bands of the medical image are modified using PN sequences depending upon the content of the corresponding 1D image vector to be embedded.

e. Four sub bands including modified sub bands are combined to generate the watermarked image using IDWT twice.

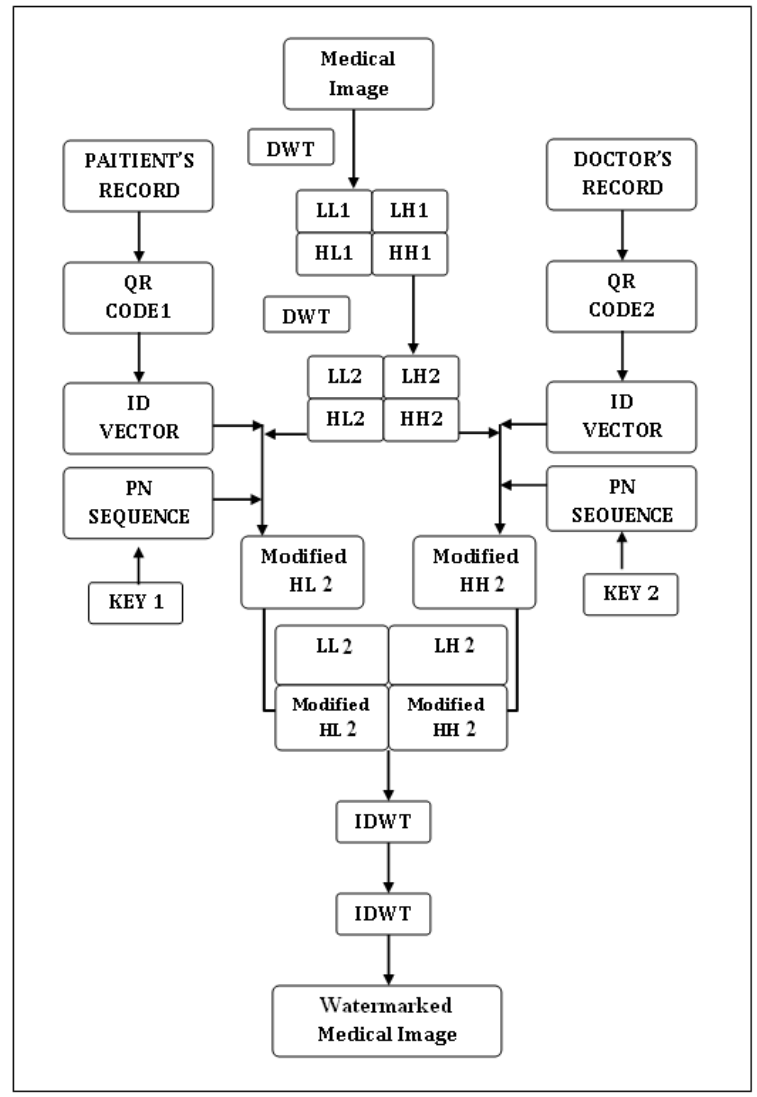

Fig 4: QR Code Hiding Process

\subsection{QR Code Extractions}

a. Size of the QR Codes and Session keys are sent to the actual receiver via a private communication channel.

b. QR Codes are recovered from the Watermarked image by knowing the size of the QR Codes and using correlation function

c. Some filters may be used to remove the unwanted signal from the QR Code.

d. Information inside the QR codes are retrieved using smart phones QR Code reader or through some standard QR reader.

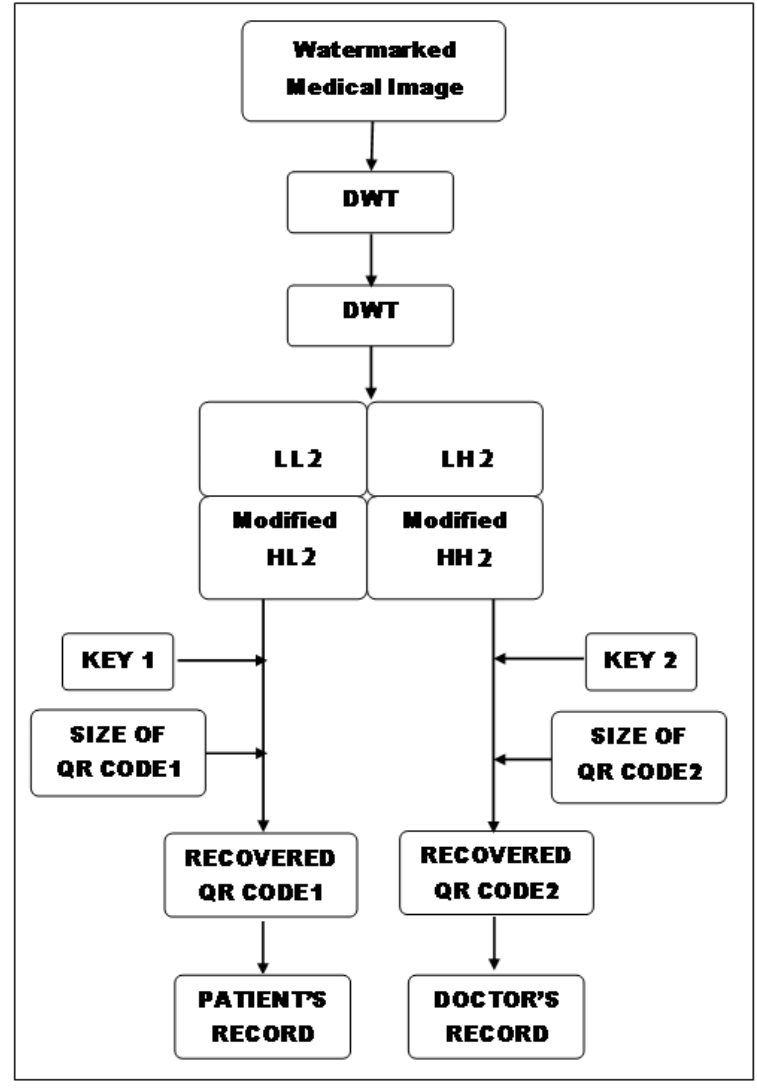

Fig 5: QR Code Extraction Process

\section{EXPLANATION OF THE ALGORITHM 3.1 QR Code Generations}

Two separate QR codes have been generated based on Patient's record and Doctor's record.

\subsection{QR Code Embedding}

Using DWT the Medical image is decomposed twice into four sub bands (LL2, LH2, HL2 and HH2). QR Codes are converted into one-dimensional vectors. Two PN sequences are generated using the session based keys and the size of any sub bands of the cover image. Bits of the QR Code vectors are embedded in HL2 and HH2 sub-bands depending upon the elements of the one-dimensional vectors and the PN sequences. The general equation used to embed the $\mathrm{QR}$ code is:

$$
\mathrm{MI}(\mathrm{x}, \mathrm{y})=\mathrm{I}(\mathrm{x}, \mathrm{y})+\mathrm{k} \times \mathrm{M}(\mathrm{x}, \mathrm{y})
$$

In which $\mathrm{I}(\mathrm{x}, \mathrm{y})$ representing the selected DWT sub band of the cover image, MI $(\mathrm{x}, \mathrm{y})$ is the modified cover image, $\mathrm{k}$ denotes the amplification factor that is usually used to adjust the invisibility of the QR Code in corresponding sub band. M $(\mathrm{x}, \mathrm{y})$ is the PN sequence. Taking all the sub bands including the modified HL2 and $\mathrm{HH} 2$ sub bands, watermarked image is obtained applying IDWT (Inverse Discrete Wavelet Transformation) twice. 


\subsection{QR Code Extraction procedure}

The Intended receiver receives the size of the QR code images and the session keys through a private and secured communication channel. Applying the same session based keys used earlier for QR Codes embedding process, the PN sequences are regenerated.

Correlation between the generated PN sequence and selected watermarked sub-band are calculated. Each correlation value is compared with the standard deviation of correlation value. If the calculated value is greater than the standard deviation value, then the extracted watermark bit will be taken as a 0 , otherwise it is taken as a 1 . The recovery process then iterates through the entire PN sequence until all the bits of the QR Code vector has been recovered. Filter may be used on recovered QR Code to remove unwanted signals.

\section{RESULTS}

This work tested with QR Codes generated from both Patient's personal information and Doctor's personal information. Smart Phone's QR Code reader has been used for extracting information from the QR Codes. Medical images are downloaded from internet, in image format, only for the sake of experiment. QR codes are generated from http://www.the-qrcode-generator.com/

The size of Doppler Medical Image is: 1024 X 1024(Gray)

The size of QR Codes is: 50 X 50(Binary)

Patient and Doctor Information

\begin{tabular}{|c|c|}
\hline Patient Record & Doctors Record \\
\hline Sirshendu Hore & Tanmay Bhattacharya \\
\hline$\underline{\text { sh@gmail.com }}$ & tb @ gmail.com \\
\hline
\end{tabular}

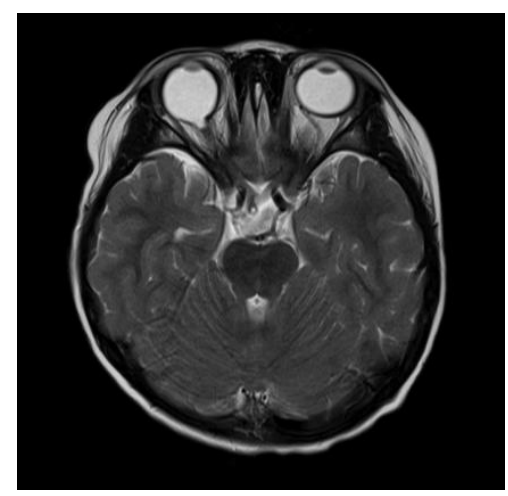

Fig. 6: Doppler Medical Image (1024X1024)

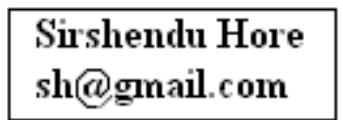

Fig. 7: Information of patient

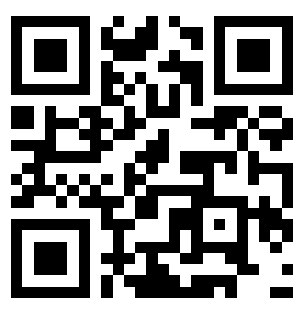

Fig. 8 Patient QR Code (50X50)

\section{Tanmay Bhattacharya tb@gmail.com}

Fig. 9: Information of Doctor

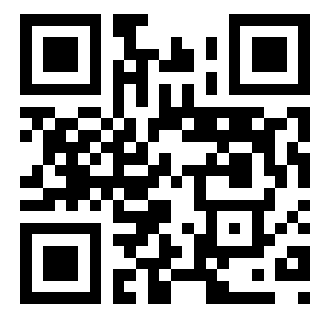

Fig. 10 Doctor QR Code (50X50)

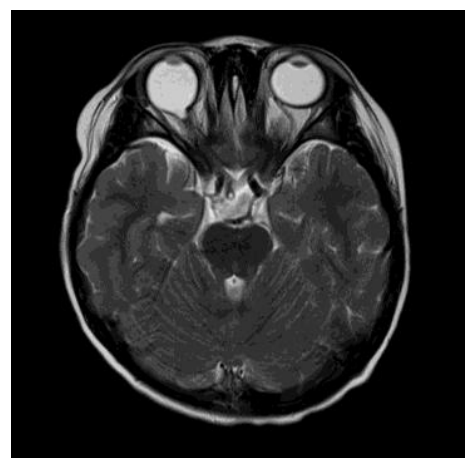

Fig. 11: Medical Doppler Watermarked Image (1024X1024)

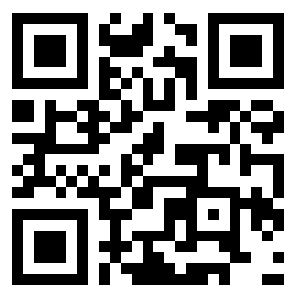

Fig. 12 Recovered Patient QR Code

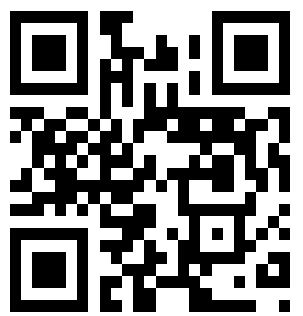

Fig. 13 Recovered Doctor QR Code 


\subsection{Robustness Test}

In Table 2 robustness test results of the proposed approach is shown.

Table 2: Sustainability against various attacks

\begin{tabular}{|c|c|c|c|}
\hline Attack & $\begin{array}{c}\text { Attacked } \\
\text { watermark } \\
\text { image }\end{array}$ & $\begin{array}{c}\text { Recovered } \\
\text { QR code } \\
\text { From HL }\end{array}$ & $\begin{array}{c}\text { Recovered } \\
\text { QR code } \\
\text { From HH }\end{array}$ \\
\hline $\begin{array}{c}\text { Noise } \\
\text { pepper) } \\
2 \%\end{array}$ \\
$\begin{array}{c}\text { Noise } \\
\text { (Gaussian) }\end{array}$
\end{tabular}

Peak Signal to Noise Ratio (PSNR): It is basically a performance metric that measures the quality of a Watermarked image. This is use to determine perceptual transparency of the Medical image with respect to Watermarked image. The cumulative squared error between the watermarked image and the original image is calculated using MSE (mean-squared error). The lower value of MSE indicates lower error rate. To compute the PSNR, MSE has been calculated using the following equation:

$$
M S E=\frac{\sum_{\mathrm{M}, \mathrm{N}}\left[I_{1}(\mathrm{~m}, \mathrm{n})-I_{2}(m, n)\right]^{2}}{M * N}
$$

Where, $\mathrm{M}$ and $\mathrm{N}$ are number of rows and columns in the input image, $I_{1}(m, n)$ is the original image and image and $I_{2}(m, n)$ is the Watermarked Image.

$$
P S N R=10 \log _{10}\left(\frac{R^{2}}{M S E}\right)
$$

$\mathrm{R}$ is the maximum fluctuation in the input image data type.

Table 3 illustrates the PSNR between Original Image and Watermarked Image.

Table 3: PSNR between Original and Watermarked Images

\begin{tabular}{|c|c|}
\hline $\begin{array}{c}\text { Cover Image } \\
\text { and }\end{array}$ & PSNR \\
\cline { 2 - 2 } Watermarked Image & 33.92 \\
\hline
\end{tabular}

Correlation coefficient: Correlation between original $\mathrm{QR}$ $\operatorname{Code}(x)$ and recovered QR Code $(y)$ has been measured by the standard linear correlation coefficient $(r)$ expression as follows:

$$
r=\frac{\sum_{i=1}^{n}\left(x_{i}-\bar{x}\right)\left(y_{i}-\bar{y}\right)}{\sqrt{\sum_{i=1}^{n}\left(x_{i}-\bar{x}\right)^{2} \sum_{i=1}^{n}\left(y_{i}-\bar{y}\right)^{2}}}
$$

Where, $x$-bar $=$ mean of $x$ and $y$-bar $=$ mean of $y$.

Table 4 illustrates the Correlation between the original QR Codes and recovered QR Codes from HL2 and HH2.

Table 4: Correlation Coefficient $(r)$ between original QR Codes and recovered QR Codes

\begin{tabular}{|c|c|c|}
\hline $\begin{array}{c}\text { Correlation } \\
\text { Coefficient }(\boldsymbol{r})\end{array}$ & from HL2 & from HH2 \\
\cline { 2 - 3 } & 0.9729 & 0.9840 \\
\hline
\end{tabular}

\section{CONCLUSION}

In the proposed method, QR codes are dispersed in the DWT sub bands of the Medical image randomly. Thus hidden information will remain unchanged after normal deformation of the Medical image and it will be difficult for any traditional image analysis method to detect the existence of the hidden information. Small visual change in between Medical image and watermarked image that is not visible by human eyes is compensated with strong security aspects. Thus this small amount of imperceptibility is quite acceptable. In the proposed approach size used for QR Code is 50X50. In future, QR codes of larger size can be used for authentication. This approach can also be extended for authentication of other medical information.

\section{REFERENCES}

[1] G. L. Friedman, "The trustworthy digital camera: Restoring credibility to the photographic image", IEEE Trans. Consumer Electron. vol. 39, pp. 905-910, Nov.1993.

[2] S.Walton, "Image Authentication for a Slippery New Age” Dr. Dobb’s J., pp. 18-26, April 1995.

[3] N.F.Johnson and S. Katzenbeisser," .A survey of steganographic techniques", in S. Katzenbeisser and F. Peticolas (Eds.): Information Hiding, pp.43-78. Artech House, Norwood, MA, 2000.

[4] Lou, D.C.and Liu, J. L. 2002. "Steganography Method for Secure Communications". Elsevier Science on Computers\& Security, 21, 5: 449-460.

[5] J.Fridrich and M. Goljan, ".Practical steganalysis of digital images-state of the art"., Proc. SPIE Photonics West, Vol. 4675, pp. 1-13, San Jose, California, Jan. 2002.

[6] Chan, C. K. and Cheng, L. M. 2003. "Hiding data in image by simple LSB substitution". Pattern Recognition, 37:469-474.

[7] Iwata, M., Miyake, K. and Shiozaki, A. 2004. "Digital Steganography Utilizing Features of JPEG Images", IEICE Transfusion Fundamentals, E87-A, 4:929-936. 
[8] Po-Yueh Chen* and Hung-Ju Lin, "A DWT Based Approach for Image Steganography", International Journal of Applied Science and Engineering 2006. 4, 3: 275-290

[9] Ali Al-Ataby and Fawzi Al-Naima, "A Modified High Capacity Image Steganography Technique Based on Wavelet Transform", The International Arab Journal of Information Technology, Vol. 7, No. 4, October 2010.

[10] Blossom Kaur, Amandeep Kaur, Jasdeep Singh, Steganographic Approach for Hiding Image in DCT Domain", International Journal of Advances in Engineering \& Technology, July 2011.

[11] Denso-Wave, "QR Code Standardization", QR Code.com. Archived from the original on 2012-09-15, Retrieved 23 April 2009.
[12] Denso-Wave. "QR Code features", Archived from the original on 2012-09-15. Retrieved $3^{\text {rd }}$ October 2011.

[13] Tanmay Bhattacharya, Nilanjan Dey and S. R. Bhadra Chaudhuri, "A Session based Multiple Image Hiding Technique using DWT and DCT", International Journal of Computer Applications (IJCA), (0975 - 8887) Volume 38 - No.5, pp 18-21, January 2012.

[14] Tanmay Bhattacharya, Sirshendu Hore and S. R. Bhadra Chaudhuri, "An Image Authentication Technique by Handwritten Signature Verification using DWT and ANN", International Journal of Computer Applications (IJCA), Volume 47- No.21, pp 35-40, 21 June 2012.

[15] Denso ADC, "QR Code Essentials", 2011, Retrieved 12 March 2013. 